\title{
УСТОЙЧИВОСТЬ И ПРИТЯЖЕНИЕ РЕШЕНИЙ НЕЛИНЕЙНЫХ СТОХАСТИЧЕСКИХ ДИФФЕРЕНЦИАЛЬНЫХ УРАВНЕНИЙ СО СТАНДАРТНЫМ И ДРОБНЫМ БРОУНОВСКИМИ ДВИЖЕНИЯМИ
}

\author{
М.М. Васьковский
}

Белгосуниверситет, факультет прикладной математики и информатики, Минск, Беларусь vaskovskii@bsu.by

Пусть заданы вероятностное пространство $(\Omega, \mathcal{F}, P)$ с потоком $\sigma$-алгебр $\left(\mathcal{F}_{t}\right), \quad r$-мерное стандартное броуновское движение $W(t), m$-мерное дробное броуновское движение $B^{H}(t)$ с показателем Херста $H \in(1 / 2,1)$ такие, что $W(t)$ является стандартным $\mathcal{F}_{t}$-броуновским движением, дробное броуновское движение $B^{H}(t)$ является $\mathcal{F}_{0}$-измеримым, процессы $W(t)$, $B^{H}(t)$ являются независимыми.

Рассмотрим нелинейную стохастическую систему

$$
d y(t)=A(t) y(t) d t+f(t, y(t)) d t+g(t, y(t)) d W(t)+b(t, y(t)) d B^{H}(t), \quad t \in R_{+}, \quad y \in R^{d},
$$

где $A: R_{+} \rightarrow R^{d \times d}-$ кусочно непрерывная функция такая, что $|A(t)| \leqslant a<\infty$ для любого $t \in R_{+}$; функции $f: R_{+} \times R^{d} \rightarrow R^{d}, g: R_{+} \times R^{d} \rightarrow R^{d \times r}, b: R_{+} \times R^{d} \rightarrow R^{d \times m}$ измеримы по Борелю, $f(t, 0)=0, g(t, 0)=0, b(t, 0)$ для почти всех $t \in R_{+}$.

Пусть $K(t, \tau)=X_{A}(t) X_{A}^{-1}(\tau)$ - матрица Коши системы

$$
d y(t)=A(t) y(t)
$$

где $X_{A}(t)=\left[x_{1}, \ldots, x_{d}\right]$ - нормальная фундаментальная матрица системы $(2)$. Через $\sigma_{\Gamma}$ обозначим показатель неправильности Гробмана системы (2). Пусть $\lambda_{i}-$ показатель Ляпунова решения $x_{i}(t)$. Не нарушая общности, считаем, что $\lambda_{1} \leqslant \ldots \leqslant \lambda_{d}$.

Обозначим через $E_{0}(\zeta)$ - условное математическое ожидание случайной величины $\zeta$ относительно $\sigma$-алгебры $\mathcal{F}_{0}$, а через $\mathcal{P}$ - множество всех $\mathcal{F}_{0}$-измеримых случайных величин $\eta: \Omega \rightarrow R^{d}$.

(A): отображения $f, g$ удовлетворяют локальному условию Липшица и имеют линейный порядок роста, отображение $b$ удовлетворяет $(\nu, 1)$-условию Гёльдера, где $\nu>1-H$.

(B1): $\lambda_{d}<0$;

(B2): существует постоянная $L$ такая, что для любых $(t, y) \in R_{+} \times R^{d}$ выполняется неравенство $\left|X_{A}^{-1}(t) f(t, y)\right|+\left|X_{A}^{-1}(t) g(t, y)\right| \leqslant L|y|$;

(B3): существуют постоянные $L, N \geqslant 0$ такие, что для любых $s, \tau \in R_{+}, y_{1}, y_{2} \in R^{d}$ выполняются неравенства $\left|X_{A}^{-1}(s) b\left(s, y_{1}\right)-X_{A}^{-1}(\tau) b\left(\tau, y_{2}\right)\right| \leqslant L\left|y_{1}-y_{2}\right|+N \sum_{i=1}^{k}|s-\tau|^{\rho_{i}}$, где $\rho_{i} \in(1-H, 1], \quad i=1, \ldots, k, \quad \rho=\max _{i} \rho_{i}>\min \{1 / 2, \nu\}$

(B4): выполняется условие В3 с постоянной $N$, равной нулю.

Для каждых $\alpha \in(0,1 / 2), T>0$ определим пространство $\mathcal{H}_{\alpha, T}$ измеримых функций $h:[0, T] \rightarrow R^{d}$ с нормой $\|h(\cdot)\|_{\mathcal{H}_{\alpha, T}}:=\sup _{t \in[0, T]}|h(t)|_{\alpha}<\infty$, где

$$
|h(t)|_{\alpha}=|h(t)|+\int_{0}^{t \in[0, T]} \frac{|h(t)-h(s)|}{(t-s)^{\alpha+1}} d s .
$$

Обозначим $\left.\alpha^{*}=\min \{\nu, \rho, 1 / 2\}\right)$.

Определение 1. Решением уравнения (1) с начальным условием $y(0)=\xi \in \mathcal{P}$ будем называть $\mathcal{F}_{t}$-согласованный процесс $y(t), t \in R_{+}$, имеющий почти наверное непрерывные по Гёльдеру траектории любого порядка $\alpha \in\left(1-H, \alpha^{*}\right)$ (т. е. функции $y(t), t \in[0, T]$, 
принадлежат пространству $\mathcal{H}_{\alpha, T}$ для любых $T \in R_{+}, \quad \alpha \in\left(1-H, \alpha^{*}\right)$ п. н.) такой, что для любых $T>0, \alpha \in\left(1-H, \alpha^{*}\right), p \geqslant 2$ с вероятностью 1 выполняется условие

$$
\int_{0}^{T} E_{0}\left(|y(t)|_{\alpha}^{p}\right) d t<\infty
$$

и для каждого $t \in R_{+}$почти наверное выполняется равенство

$$
y(t)=K(t, 0) \xi+\int_{0}^{t} K(t, s) f(s, y(s)) d s+\int_{0}^{t} K(t, s) g(s, y(s)) d W(s)+\int_{0}^{t} K(t, s) b(s, y(s)) d B^{H}(s),
$$

где интеграл по стандартному броуновскому движению - интеграл Ито, интеграл по дробному броуновскому движению - потраекторный интеграл Римана - Стилтьеса.

Определение 2. Будем говорить, что решение $y(t)$ уравнения (1) с начальным условием $y(0)=\xi \in \mathcal{P}$ является единственным, если для любого решения $z(t)$ уравнения (1) с начальным условием $z(0)=\xi$ выполняется условие $P\left(y(t)=z(t) \forall t \in R_{+}\right)=1$.

Определение 3. Пусть $\alpha \in(1-H, 1 / 2), \quad p \geqslant 1$. Будем говорить, что нулевое решение $y(t) \equiv 0$ уравнения (1) является $(\alpha, p)$-асимптотически устойчивым по вероятности, если выполняются следующие условия: 1$)$ для любых $\varepsilon_{1}, \varepsilon_{2}>0$ существует постоянная $r=r\left(\varepsilon_{1}, \varepsilon_{2}\right)>0$ такая, что для любого $\xi \in \mathcal{P},|\xi| \leqslant r$ п. н., выполняется неравенство $P\left(E_{0}\left(\left|y_{\xi}(t)\right|_{\alpha}^{p}\right)>\varepsilon_{1}\right) \leqslant \varepsilon_{2}$ для любых $\left.t \in R_{+}, 2\right)$ для любого $\varepsilon>0$ существует постоянная $K=K(\varepsilon)>0$ такая, что для любого $\xi \in \mathcal{P},|\xi| \leqslant K$ п. н., имеет место сходимость $P\left(E_{0}\left(\left|y_{\xi}(t)\right|_{\alpha}^{p}\right)>\varepsilon\right) \rightarrow 0$ при $t \rightarrow+\infty$, где $y_{\xi}(t)-$ решение уравнения (1) с начальным условием $y(0)=\xi$.

Определение 4. Пусть $\alpha \in(1-H, 1 / 2), p \geqslant 1$. Будем говорить, что нулевое решение уравнения (1) является $(\alpha, p)$-притягивающим, если для любых $\varepsilon>0, K>0$ и любой случайной величины $\xi \in \mathcal{P},|\xi| \leqslant K$ п.н., имеет место сходимость $P\left(E_{0}\left(\left|y_{\xi}(t)\right|_{\alpha}^{p}\right)>\varepsilon\right) \rightarrow 0$ при $t \rightarrow+\infty$, где $y_{\xi}(t)-$ решение уравнения (1) с начальным условием $y(0)=\xi$.

Предложение 1. [1]. Если выполняется условие $A$, то для любого $\xi \in \mathcal{P}$ уравнение (1) с начальным условием $y(0)=\xi$ имеет единственное решение.

Теорема 1. Пусть выполняются условия A, B1, В2, В4, тогда для любых $\alpha \in\left(1-H, \alpha^{*}\right)$, $p \geqslant p^{*}(\alpha, H)=\max \{4 /(1-2 \alpha), 1 /(1-H)\}$ нулевое решение уравнения (1) является $(\alpha, p)$ асимптотически устойчивым по вероятности.

Теорема 2. Пусть выполняются условия A, B1, В2, В3, тогда для любых $\alpha \in\left(1-H, \alpha^{*}\right)$, $p \geqslant p^{*}(\alpha, H)$ нулевое решение уравнения (1) является $(\alpha, p)$-притягивающим.

\title{
Литература
}

1. Леваков А.А., Васьковский М. М. Существование решений стохастических дифференииальных включений со стандартным и дробным броуновскими движениями // Дифференц. уравнения. 2015. T. 51. № 8. C. 997-1003.

\section{ПРИМЕНЕНИЕ ДИФФЕРЕНЦИАЛЬНЫХ УРАВНЕНИЙ К РАСЧЕТУ ПЛЕНОЧНЫХ ТЕЧЕНИЙ}

\author{
А.М. Волк \\ Белорусский государственный технологический университет \\ anatoliyvolk@mail.ru
}

Пленочные течения широко используются в газожидкостных реакторах, тепломассообменных аппаратах и других технических устройствах [1-4]. Гидродинамика этих течений 